\title{
Modifications, du travail de sortie des surfaces clivées de GaAs, liées au refroidissement
}

\author{
J. Bonnet, L. Soonckindt et L. Lassabatère \\ Laboratoire d'Etudes des Surfaces, Interfaces et Composants (*) \\ U.S.T.L., place Eugène Bataillon, 34060 Montpellier Cedex, France
}

(Reçu le 9 septembre 1983, révisé le 7 février 1984, accepté le 22 mars 1984)

\begin{abstract}
Résumé. - Nous étudions l'évolution des topographies du travail de sortie de surfaces de GaAs de type n, obtenues par clivages sous ultra-vide à $400 \mathrm{~K}$ ou à l'ambiante, lorsque l'échantillon est refroidi jusqu'au voisinage de la température de l'azote liquide. Nous mettons en évidence un effet irréversible important. Une manipulation originale nous permet de proposer une interprétation reposant sur l'interaction de la surface avec les molécules de gaz résiduels et d'expliquer certains résultats anciens en désaccord avec ceux obtenus actuellement sur les surfaces de GaAs de type $n$.
\end{abstract}

\begin{abstract}
After cleavage at room temperature, $n$ GaAs topographic work functions are studied when the samples are cooled down to liquid nitrogen temperature. We set off an important irreversible effect. An original experiment enables us to give an interpretation based on the interaction of the surface with residual gaz molecules. This permits to explain previous experiments whose results strongly differ from those obtained now on $\mathrm{n}$ GaAs surfaces.
\end{abstract}

\section{Introduction.}

L'étude des propriétés physiques des matériaux, comme des composants électroniques, peut nécessiter des expérimentations dans une large gamme de températures. Dans ces expériences, la température est utilisée comme un paramètre permettant l'étude d'un mécanisme donné dont les propriétés ne sont pas altérées par un cycle en température, ou comme un paramètre induisant des modifications des propriétés liées au traitement thermique.

A titre d'exemple, les études de transport en volume rentrent dans la première catégorie, les études de modifications de structure ou de composition de surface dans la deuxième catégorie.

S’il est vrai que, dans les travaux sur les surfaces, la température est utilisée essentiellement pour nettoyer, restructurer les surfaces à étudier, certains chercheurs l'ont cependant utilisée comme paramètre pour déterminer des caractéristiques physiques intrinsèques. Dans cet esprit, en 1971, Peter Mark proposait une méthode axée sur la mesure des variations de barrière de surface avec la température [1], pour

(*) E.R.A. no 1022, associée au C.N.R.S.

JoURNAL DE PHYSIQUE. - T. 45, № 7, JUILLET 1984 déterminer les positions énergétiques des états de surface. Cette idée a été reprise et développée par L. K. Galbraith et T. E. Fisher [2] au cours des années 1971-72 et plus récemment par le groupe Mönch [3] et le nôtre $[4,5]$. Si les études théoriques de modélisation nécessaires à l'interprétation des résultats expérimentaux ne posent pas de problème majeur [6], l'expérimentation, délicate, conduit à se poser la question suivante : dans quelle mesure le traitement en température ne modifie-t-il pas la surface et n'introduit-il pas des effets parasites se superposant à l'effet que l'on souhaite étudier et pouvant le masquer ?

Répondre à cette question est impératif, avant toute interprétation des résultats expérimentaux.

L'étude que nous présentons a pour objet d'illustrer ce point et de mettre en garde sur des interprétations trop rapides. Partant d'une surface de GaAs clivée dont nous étudions le travail de sortie par la méthode de Kelvin, nous montrons, à l'aide de relevés topographiques, que la zone de l'échantillon située en face de l'électrode de mesure a un comportement différent de celui des zones qui l'entourent.

Bien que le champ électrique soit nul entre l'électrode et la surface, l'électrode a donc une action et on peut a priori envisager qu'elle induit des modifi- 
cations de propriété de la surface de l'échantillon situé en face d'elle ou qu'inversement elle la protège, lui conservant ainsi ses propriétés intrinsèques.

Dans cet article, nous rapportons les résultats expérimentaux clefs qui permettent de préciser ces points, résultats dont la prise en compte peut, d'une part, amener à reconsidérer en termes nouveaux les interprétations et conclusions concernant GaAs publiées ces quinze dernières années et, d'autre part, conduire les expérimentateurs utilisant les basses températures pour les études en surface, à bien s'assurer de la signification des mesures effectuées.

\section{Dispositif expérimental. Méthodologie.}

L'ensemble expérimental comprend :

- Une enceinte munie d'une pompe ionique (dont le vide limite se situe aux environs de $7 \times 10^{-11}$ torr), d'une jauge à ionisation Bayard Alpert, d'un équipement d'étude de diffraction d'électrons lents et de spectroscopie Auger.

- Un cliveur porte-échantillon dont la température $T$, mesurée par une résistance au platine, peut être ajustée entre $85 \mathrm{~K}$ et $400 \mathrm{~K}$. Un dispositif de régulation rend possible la programmation de remontées linéaires de la température avec le temps ainsi que de paliers de température.

- Une sonde de Kelvin qui permet le relevé de topographies du travail de sortie et du photovoltage de surface. Cette sonde, constituée par une électrode d'or hémisphérique, d'un diamètre voisin de $2 \mathrm{~mm}$, se déplace parallèlement à la surface à étudier, à une distance $d$ qui doit être maintenue constante pour que la mesure soit reproductible $(d \simeq 20 \pm 1 \mu \mathrm{m})$. L'asservissement [7] que nous avons réalisé dans ce but permet en outre, lorsque l'électrode a été écartée, de la repositionner avec une grande précision.

La méthodologie est la suivante : les échantillons de GaAs, qui se présentent sous forme de barreaux parallélépipédiques de section droite $4 \times 8 \mathrm{~mm}^{2}$ sont clivés à différentes températures, à une pression comprise entre $10^{-10}$ et $7 \times 10^{-11}$ torr. La pression est mesurée avant clivage par une jauge Bayard Alpert qui est ensuite maintenue éteinte pendant toute la manipulation pour éviter d'éventuelles perturbations. Un relevé topographique du travail de sortie (Fig. 1) est effectué après clivage; l'évolution de ces topographies est également suivie lors des études thermiques (refroidissement ou réchauffement).

\section{Résultats.}

3.1 EVOLUTION DU TRAVAIL DE SORTIE AVEC LA TEMPÉRATURe. EFFET INDUIT PAR L'ÉleCtrode. - Après clivage à température ambiante nous avons relevé les variations topographiques du travail de sortie $\phi$ en nous déplaçant de A vers B (Fig. 2, courbe 1). La

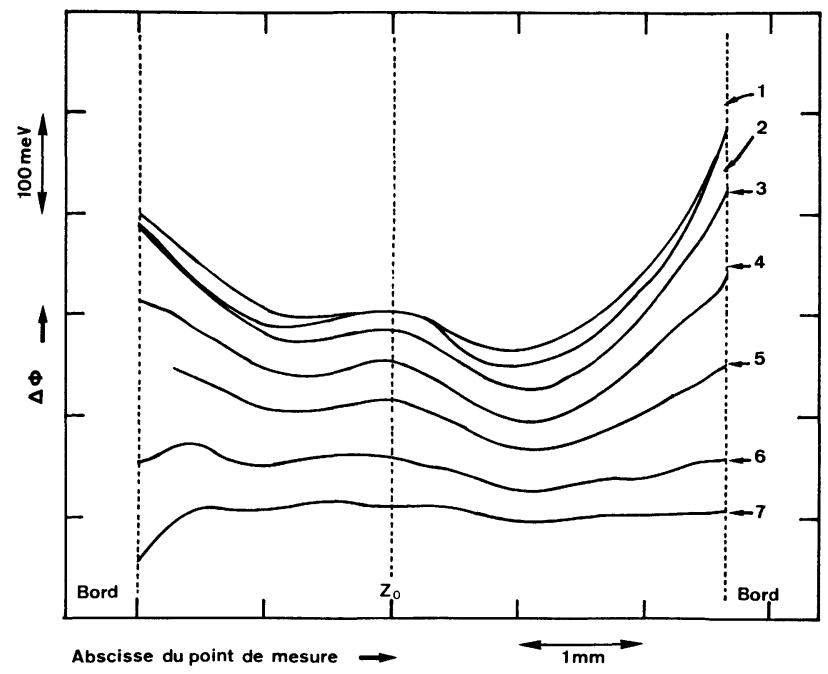

Fig. 1. - Evolution du travail de sortie d'une surface de GaAs $n$ obtenue par clivage sous ultravide à $400 \mathrm{~K}$, lors d'un abaissement de la température $T$. $1-T=400 \mathrm{~K}$ (clivage), $2-T=335 \mathrm{~K}, 3-T=265 \mathrm{~K}, 4-T=205 \mathrm{~K}$, $5-T=165 \mathrm{~K}, 6-T=110 \mathrm{~K}, 7-T=85 \mathrm{~K}$.

[Variations during cooling of the work function of $\mathrm{n} \mathrm{GaAs}$ cleaved at $T=400 \mathrm{~K}$.]

surface n'est pas uniforme et présente des états de surface induits par des défauts, ce qui entraîne d'importantes variations de $\phi$. Arrivé en B et l'électrode étant maintenue en ce point à une distance $d_{0}$ de la surface égale à celle adoptée pour le relevé de la topographie précédente, nous avons alors abaissé la température jusqu'à $85 \mathrm{~K}$ puis effectué un nouveau relevé en allant de B vers A (Fig. 2, courbe 2). La comparaison des deux courbes montre que les variations du travail de sortie en $B$ sont faibles et très inférieures à celles obtenues pour d'autres points de la surface. En particulier, le travail de sortie au point $C$, identique à l'ambiante à celui de $\mathrm{B}$, est maintenant nettement différent. Il apparaît donc très clairement que la présence de l'électrode en face de la surface réduit les variations du travail de sortie induites par le refroidissement. Ce résultat est confirmé par la figure 2, courbe 3 obtenue après que la position de l'électrode ait été changée de façon à découvrir la surface au point B. Comme le champ électrique entre la surface et l'électrode est maintenu égal à zéro, l'effet induit par celle-ci ne peut être électrique. Il faut donc envisager un effet éventuel d'un éclairement parasite ou de gaz résiduels. Ceci conduit à effectuer une étude complémentaire pour préciser le rôle de l'électrode et analyser les effets induits par le refroidissement.

\section{2 EFFET DE L'ÉVOLUTION DE $\phi$ (ÉLECTRODE ÉCARTÉE).}

3.2.1. Conditions de mesure. - Après clivage, nous avons étudié dans l'obscurité l'évolution des topographies de $\phi$ en fonction de la température pour un échantillon de GaAs de type $\mathrm{n}\left(n \simeq 5 \times 10^{17} \mathrm{~cm}^{-3}\right)$. 


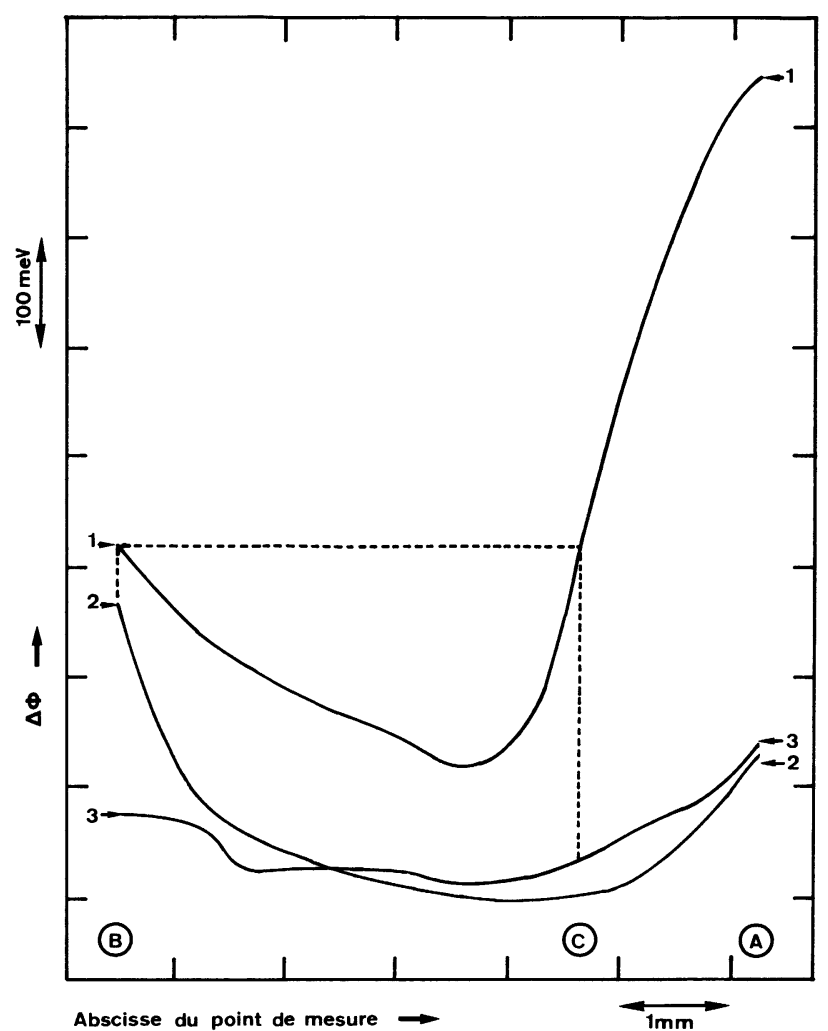

Fig. 2. - Evolution du travail de sortie d'une surface de GaAs n obtenue par clivage sous ultravide. 1 - après clivage à $300 \mathrm{~K}, 2$ - après abaissement de la température à $85 \mathrm{~K}$, l'électrode ayant été maintenue en position de mesure à l'abscisse $\mathrm{B}, 3$ - relevé à $85 \mathrm{~K}$ succédant à un écartement de l'électrode à l'abscisse $\mathrm{B}$.

[Work function variations of $\mathbf{n}$ GaAs cleaved under UHV. 1 - after cleavage at $T=300 \mathrm{~K}, 2$ - after cooling at $T=85 \mathrm{~K}$, the probe being kept close to the sample at the abscissa $\mathrm{B}$, 3 - at $T=85 \mathrm{~K}$, after the probe has been moved away from the sample at the abscissa $B$.]

Entre des mesures à différentes températures l'électrode de mesure était écartée de la surface semiconductrice puis rapprochée au moment de la mesure.

3.2.2. Effet du refroidissement. - L'étude des courbes de la figure 1 met en évidence des variations importantes lors du refroidissement. La valeur de $\phi$ diminue sur toute la surface, l'effet étant plus important sur les zones mal clivées que sur les zones bien clivées. Les inhomogénéités initiales sont atténuées. A $85 \mathrm{~K}$ le travail de sortie est pratiquement uniforme pour les différentes zones de la surface (Fig. 1, courbe 7).

3.2.3. Effet $d u$ réchauffement. -- Le réchauffement jusqu'à $T \simeq 300 \mathrm{~K}$ ne restaure que très partiellement les topographies initiales (Fig. 3). Si les inhomogénéités initiales réapparaissent, leur amplitude est beaucoup plus faible. Les courbes sont plus plates et, dans les mêmes conditions de température, la valeur moyenne du travail de sortie a augmenté. Ceci apparaît nettement sur la figure 4 qui retrace les variations de $\phi$ en fonction de la température pour le point de

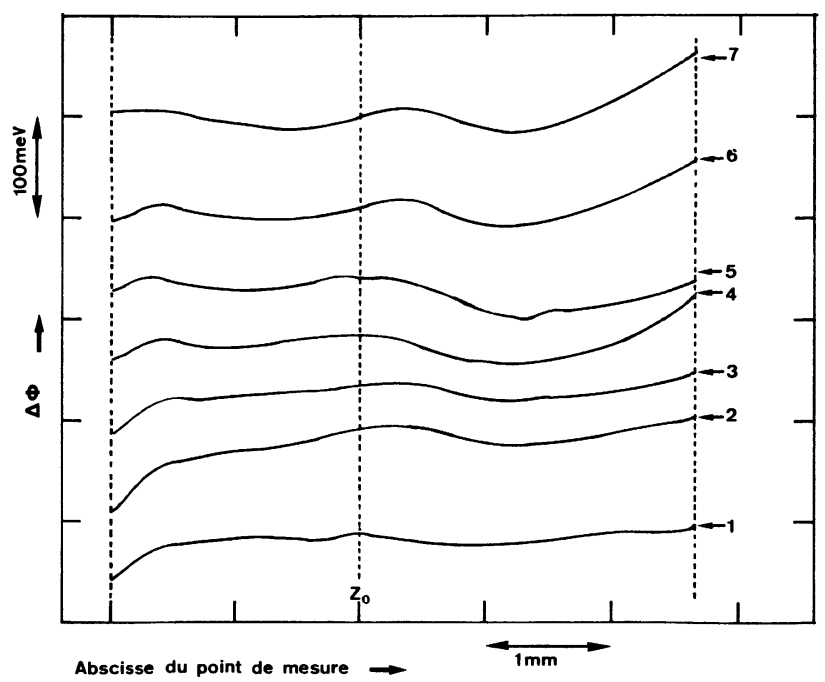

Fig. 3. - Evolution du travail de sortie de la surface de GaAs n présentée figure 1, lors de la remontée en température. $1-T=85 \mathrm{~K}, 2-T=125 \mathrm{~K}, 3-T=130 \mathrm{~K}, 4-T=160 \mathrm{~K}$, $5-T=180 \mathrm{~K}, 6-T=220 \mathrm{~K}, 7-T=280 \mathrm{~K}$.

[Variations during warming of the work function of the n GaAs sample studied figure 1.]

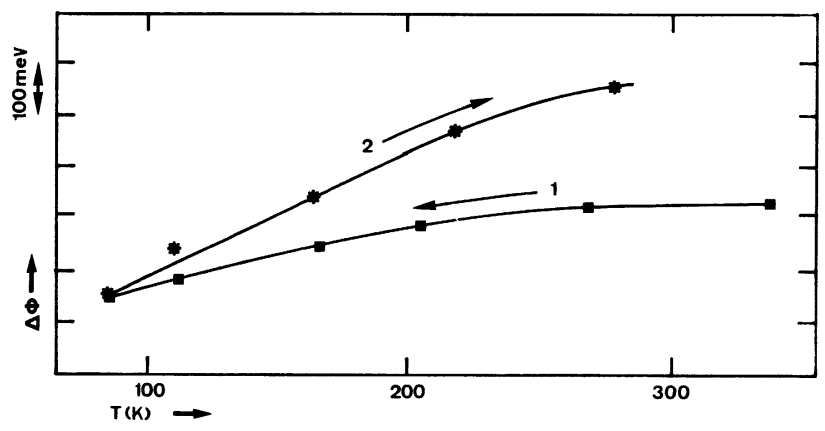

Fig. 4. - Evolution du travail de sortie du point d'abscisse $Z_{0}$ des figures 1 et 3 lors du cycle descente (courbe 1)remontée (courbe 2) en température.

[Work function variations of the point of abscissa $Z_{0}$ (see figs. 1 and 3) while cooling (curve 1) and warming (curve 2).]

la surface d'abscisse $Z_{0}$. Aux environs de $300 \mathrm{~K}$ le photovoltage de surface est pratiquement uniforme sur toute la surface. Son signe indique que la courbure des bandes est négative : la surface est en déplétion.

En conclusion, le fait de refroidir l'échantillon a modifié profondément et de façon irréversible les propriétés électroniques de la surface; en particulier, une barrière est apparue sur les zones qui en étaient initialement dépourvues.

Cette tendance à l'uniformisation se confirme si on effectue des cycles successifs de refroidissementréchauffement ou si on augmente le vide résiduel ou la durée du refroidissement. Les travaux de sortie des zones bien et mal clivées deviennent identiques. On retrouve là un effet semblable à celui induit par 
l'adsorption de gaz [8] ou le dépôt de faibles couches métalliques sur surfaces clivées, effet qui s'explique dans ce cas en termes de défauts induits et d'ancrage du niveau de Fermi.

3.2.4. Variations de $\phi$ en fonction de la température après uniformisation de la surface. - L'électrode étant maintenue à distance constante de la surface, en position de mesure, on effectue un cycle refroidissement-réchauffement entre $300 \mathrm{~K}$ et $85 \mathrm{~K}$. Les variations sont pratiquement linéaires et réversibles (Fig. 5).

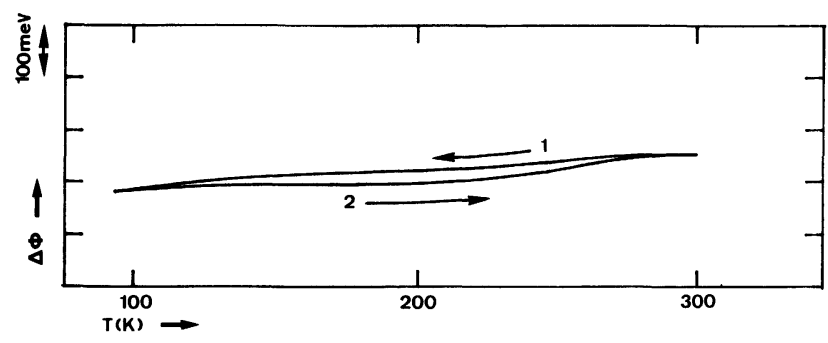

Fig. 5. - Evolution du travail de sortie en fonction de la température en un point d'une surface de $\mathrm{GaAs} \mathbf{n}$ ayant subi l'effet irréversible lors d'un premier refroidissement. Durant le relevé, l'électrode est maintenue en permanence en position de mesure. 1 - refroidissement, 2 - réchauffement.

[Work function variations versus temperature of one point of an $\mathrm{n} \mathrm{GaAs}$ surface which has been submitted to the irreversible effect after cooling. During the measurement the probe is continuously kept close to the sample. 1 - cooling, 2 - warming.]

3.3 EFFET DE L'ÉCARTEMENT DE L'ÉLECTRODE. - La surface étant uniformisée, comme indiqué paragraphe 3.2 .3 et à la température de $300 \mathrm{~K}$, on refroidit à nouveau l'échantillon à $85 \mathrm{~K}$. L'électrode qui avait été maintenue en place lors de la descente en température est écartée quelques instants, puis remise en position de mesure. On constate alors une variation importante de $\phi$. La courbe de remontée en température (Fig. 6) est différente de celle obtenue sans écarter l'électrode. La valeur finale de $\phi$ à l'ambiante est cependant la même que celle obtenue avant refroidissement. On a donc un effet réversible induit par l'écartement de l'électrode qui se superpose à l'effet irréversible précédemment décrit.

Interprétation.

Les résultats précédents ont mis en évidence le rôle particulier joué par l'électrode, lors de la descente en température de l'échantillon. On peut envisager plusieurs hypothèses plausibles pour expliquer les résultats obtenus et entre autres :

- une action électrique éventuelle de l'électrode résultant de la différence de potentiel entre la surface et l'électrode : cette hypothèse est à exclure car on travaille ici en compensant de façon automatique la différence de potentiel de contact entre la surface et l'électrode ;

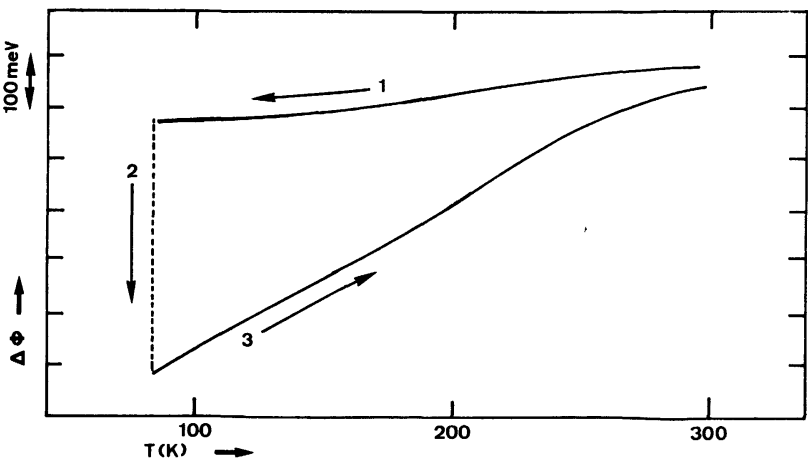

Fig. 6. - Evolution du travail de sortie en fonction de la température en un point d'une surface de GaAs $n$ ayant subi l'effet irréversible lors d'un premier refroidissement. 1 refroidissement (électrode en position de mesure), 2 - électrode écartée pendant $10 \mathrm{~min}$. puis remise en position de mesure, 3 - réchauffement (électrode en position de mesure).

[Work function variations versus temperature of one point of an $\mathrm{n} \mathrm{GaAs}$ surface which has been submitted to the irreversible effect after a first cooling. 1 - cooling (the probe is kept close to the sample when cooling), 2 - the probe is moved away from the sample for $10 \mathrm{~min}$. then brought closer to the sample for measurement, 3 - warming (the probe is kept close to the sample for measurement).]

- une possible photoadsorption ou photodésorption : travaillant dans l'obscurité, cette deuxième éventualité est également à exclure ;

- l'arrivée sur la surface de particules chargées, provenant de la pompe ionique : une électrode disposée dans le bâti au voisinage de l'échantillon, polarisée de façon à attirer d'éventuelles charges conduit à exclure cette nouvelle hypothèse; le courant mesuré est beaucoup trop faible pour permettre d'expliquer les variations observées et les cinétiques;

- une adsorption de gaz résiduel, l'électrode lorsqu'elle est écartée permettant à un plus grand nombre d'atomes ou de molécules d'atteindre la surface et de s'y fixer. Cette dernière hypothèse est plausible.

En effet, le nombre de molécules heurtant la surface par unité d'aire et de temps est donné par :

$$
n=\frac{p}{\sqrt{2 \pi m k T}}=n_{0} \times p
$$

où $m$ est la masse des molécules, $p$ la pression, $T$ la température. A la température ambiante et pour l'oxygène ceci conduit à :

$$
n_{0}=3,48 \times 10^{14} \text { molécules } \times \mathrm{cm}^{-2} \times \mathrm{L}^{-1} .
$$

A la pression résiduelle qui est celle des bâtis à ultravide (de l'ordre de $7 \times 10^{-11}$ torr), il tombe donc par seconde et par $\mathrm{cm}^{2}$

$$
n=n_{0} \times p=2,4 \times 10^{10} \text { molécules . }
$$

Le temps pendant lequel, dans l'expérience correspondant aux résultats rapportés (Fig. 3), l'électrode 
métallique est restée au point $\mathrm{B}$ est de l'ordre de la dizaine de minutes. Le nombre de molécules tombant sur la surface pendant ce laps de temps est donc de l'ordre de $1,24 \times 10^{13} \times \mathrm{cm}^{-2}$.

$\mathrm{Si}$ on prend en compte le fait que l'écartement de l'électrode s'est traduit par la formation d'une barrière de surface sur les zones bien clivées, on est conduit à associer aux particules incidentes des états électroniques chargés dont la densité nécessaire pour expliquer la formation d'une barrière de l'ordre de plusieurs centaines de $\mathrm{mV}$ se situe aux alentours de $10^{12}-10^{13} \times \mathrm{cm}^{-2}$.

Cette densité d'état, du même ordre que le nombre de molécules tombées sur la surface lorsque l'électrode est écartée, lui est cependant légèrement inférieure, ce qui revient à dire que toutes les molécules incidentes ne contribuent pas à la formation d'un état électronique chargé.

Ceci pourrait s'expliquer :

1) par un coefficient de collage à $85 \mathrm{~K}$ voisin de 1 , valeur nettement supérieure à celles obtenues à l'ambiante pour l'oxygène et échelonnées de $10^{-9}$ à $10^{-2}[9,10]$ mais qui est cependant acceptable si on prend en compte dans l'expression qui donne le coefficient de collage le terme exponentiel de forme $\exp =\left[-\frac{E_{\mathrm{a}}}{k T}\right]$ où $E_{\mathrm{a}}$ est l'énergie d'activation;

2) par la création d'états dont la position énergétique serait telle que certains d'entre eux seulement seraient chargés. L'uniformisation du travail de sortie résultant d'une exposition suffisante de la surface serait due dans cette hypothèse à la création d'une densité d'états accepteurs suffisante pour ancrer le niveau de Fermi, densité qui suivant la position énergétique de l'état se situe entre $10^{12}$ et $10^{15} \times \mathrm{cm}^{-2}$.

L'effet de l'électrode correspond donc à une protection de la surface contre les chocs de particules, protection que conforte le calcul du nombre de particules pouvant heurter la surface à étudier lorsque l'électrode est en face d'elle. Dans les conditions de la mesure (diamètre de l'électrode $2 \mathrm{~mm}$, distance électrode-surface $20 \mu \mathrm{m}$ ) nous avons calculé la densité de molécules atteignant respectivement le centre A ou le bord B de la surface couverte. Nous avons obtenu :

$$
n_{1 \mathrm{~A}} \simeq 10^{-4} \mathrm{n} ; \quad n_{1 \mathrm{~B}} \simeq 0,5 \mathrm{n} .
$$

Ceci conduit à un nombre total de chocs sur la surface protégée très inférieur à ce qu'il serait sans la présence de l'électrode et explique que les modifications des propriétés électriques sous l'électrode soient très fortement diminuées.

\section{Conclusion.}

Les résultats que nous avons rapportés illustrent le rôle qu'a joué la présence de l'électrode en face de la surface à étudier et montrent que lors d'études à basse température les conditions expérimentales peuvent introduire des effets parasites importants. Dans le cas de GaAs en particulier, l'étude des propriétés intrinsèques de la surface ne peut être effectuée que si on est certain que la surface est bien protégée de façon à réduire ou mieux, exclure, tout effet induit. Dans le cas contraire on peut, par erreur, caractériser un effet extrinsèque tout en croyant étudier un effet intrinsèque. Il peut en être ainsi par exemple pour les états électroniques intrinsèques : une surface bien clivée ne présente pas d'états dans la bande interdite; les variations du travail de sortie, quand on descend en température devraient donc traduire les variations d'affinité électronique et de position du niveau de Fermi. Or certains résultats expérimentaux obtenus à basse température mettant en évidence des variations beaucoup plus importantes, ont été interprétés en termes d'états [2].

L'importance de l'effet induit, mis en évidence par mesure du travail de sortie vrai, c'est-à-dire tel qu'il est sur la portion de surface de l'échantillon située en regard de l'électrode, dépend de la probabilité de la création d'un état lors de l'interaction moléculesurface lorsque celle-ci est découverte et de la position énergétique de l'état. Elle est donc spécifique du couple matériau-gaz. Ceci permet d'expliquer que pour de nombreux matériaux, comme GaSb par exemple, la descente en température ne se traduise pas par des modifications semblables du travail de sortie. L'interaction peut en effet ne pas créer des états ou créer des états dont la position est telle qu'ils ne modifieront pas la barrière. Ceci ne veut cependant pas dire qu'il faille exclure une modification induite, modification ne se traduisant pas par des variations du travail de sortie et que d'autres méthodes pourraient permettre de mettre en évidence et caractériser.

En conclusion donc, il faut souligner la prudence avec laquelle on doit aborder les études de surfaces à basse température et les précautions qui doivent précéder toute interprétation.

\section{Bibliographie}

[1] Mark, P., Surf. Sci. 25 (1971) 192.

[2] Galbraith, L. K. and Fischer, T. E., Surf. Sci. 30 (1972) 185.

[3] Monch, W., Clemens, H. J., J. Vac. Sci. Technol. 16 (5) (1979) 1238.

[4] Bonnet, J., Soonckindt, L. et Lassabatère, L., Surf. Sci. 91 (1980) 283.

[5] Bonnet, J., Soonckindt, L. et Lassabatère, L., Thin Solid Films 91 (1982) 283.
[6] Davidson, S. G., Levine, J. D., Solid State Physics 25 (1970) 1.

[7] Bonnet, J., SOONCKIndt, L. et LASSABatère, L., Congrès MECO 77, juin 1977, Zurich (Suisse).

[8] SoOnCKindt, L., Bonnet, J. et Lassabatère, L., Thin Solid Films 78 (1981) 167.

[9] Pianetta, P., Lindau, I., Gregory, P. E., Garner, C. M., SPICER, W. E., Surf. Sci. 72 (1978) 298.

[10] Proix, F., Houzay, F., J. Phys. C 13 (1980) 1845. 